\title{
Semiconductor detectors for high-luminosity environments
}

\author{
Paula Collins \\ PH Department, CERN, CH1211, Geneve 23, Switzerland
}

Available online 20 July 2007

\begin{abstract}
As the final touches are being put to the LHC detectors, the race is on to perfect technologies which could be used to confront the challenges of the ultra-high luminosities of the SLHC and International Linear Collider (ILC). These challenges include ever more hostile radiation environments, short signal shaping times and increasing emphasis on the highest possible granularity combined with the lowest possible mass. In the semiconductor detector field, dedicated studies have identified many mechanisms behind radiation damage and pointed the way towards the use of new techniques such as $\mathrm{MCz}$ silicon and p-type sensors. In the most extreme environments, completely new approaches, such as the use of diamond or 3D sensor detectors, will be essential. Pixel technology, which has been successfully employed to build large-scale vertex detection systems for the LHC, will be pushed towards higher density, lower mass and greater integration, to satisfy in particular the ILC requirements. We review the highlights of the current generation of semiconductor detectors and discuss some of the exciting prospects for future developments.
\end{abstract}

(C) 2007 Elsevier B.V. All rights reserved.

PACS: 29.40.-n; 61.80.-x; 29.40.Gx; 29.40.Wk

Keywords: Semiconductor; Microstrip; Pixel; Radiation hardness; SLHC; ILC; MCz; MAPS; p-type; Vertex detectors; 3D; MAPS

\section{Introduction}

Silicon strip and pixel detectors have played a crucial role in modern particle physics experiments. They are available with excellent yield from many vendors, and have an established track record of reconstructing secondary vertices in both fixed target and collider environments.

It is interesting to cast a glance backwards at the impact of silicon strip detectors at LEP, an environment with a luminosity of around $10^{31} \mathrm{~cm}^{-2} \mathrm{~s}^{-1}$. There, prototype detectors were being installed already in 1989; by 1991 all experiments had silicon vertex detectors. At the same time the move was made from $8 \mathrm{~cm}$ radius stainless steel beampipes to $5.3 \mathrm{~cm}$ beryllium versions. Operating at modest voltages, these detectors gave an excellent position resolution, and their introduction was crucial for the development of the $\mathrm{b}, \mathrm{c}$ and $\tau$ physics programmes. Moving to luminosities of around $10^{32} \mathrm{~cm}^{-2} \mathrm{~s}^{-1}$ at the Tevatron, silicon has taken on an additional role through track triggers, which identify hadronic vertices online. The

E-mail address: paula.collins@cern.ch vertexing capabilities remain vital, most prominently in the recent spectacular discovery of $B_{s}$ mixing. These achievements are illustrated in Fig. 1.

At the LHC the luminosity rises again to a nominal operating value of $10^{34} \mathrm{~cm}^{-2} \mathrm{~s}^{-1}$, and the silicon faces new challenges, principally:

- Around 23 overlapping interactions in each bunch crossing, occurring every $25 \mathrm{~ns}$, which has required the development of new front-end electronics with short signal shaping.

- Inside the tracker acceptance of the large experiments $(\eta<2.5)$ there can be 750 charged tracks per bunch crossing, and good granularity is essential for track reconstruction.

- Given the typical rates of $B$ hadron production $\left(\approx 10^{14}\right.$ per year), $\mathrm{t} \overline{\mathrm{t}}$ production $\left(\approx 10^{8}\right.$ per year $)$ as well as possible Higgs signals, above a background of $\approx 10^{16}$ inelastic collisions, the impact parameter resolution is critical to separate signal from background.

- All this is occurring in a very harsh radiation environment: the fast hadron dose at a radius of $4 \mathrm{~cm}$ over 
a

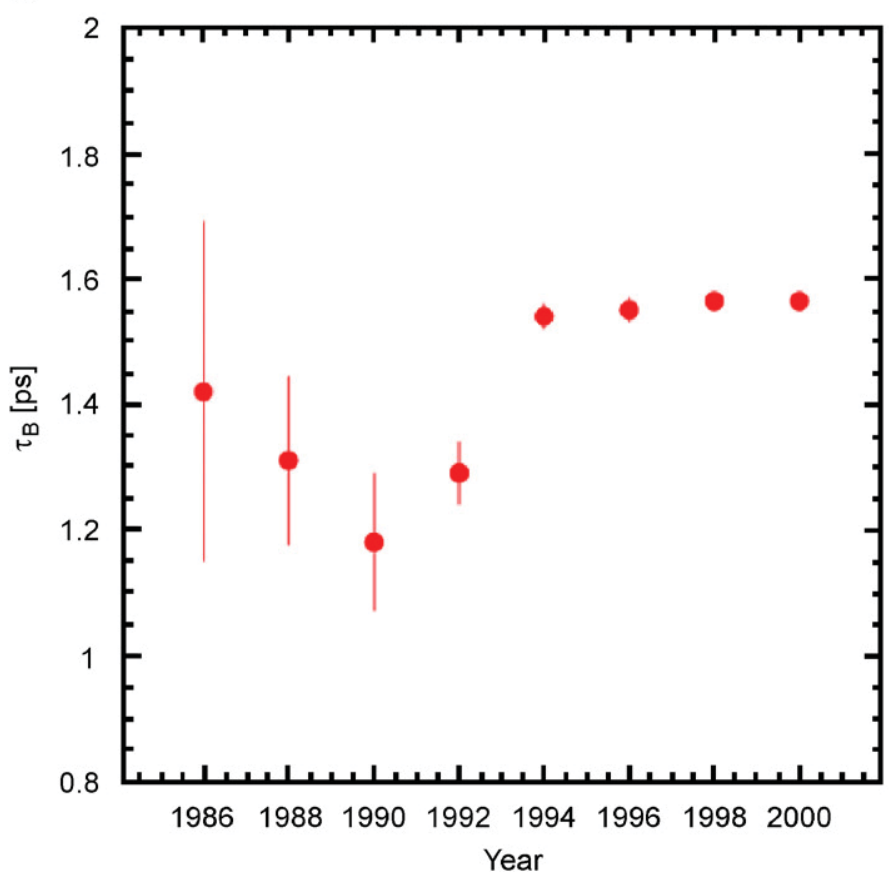

b

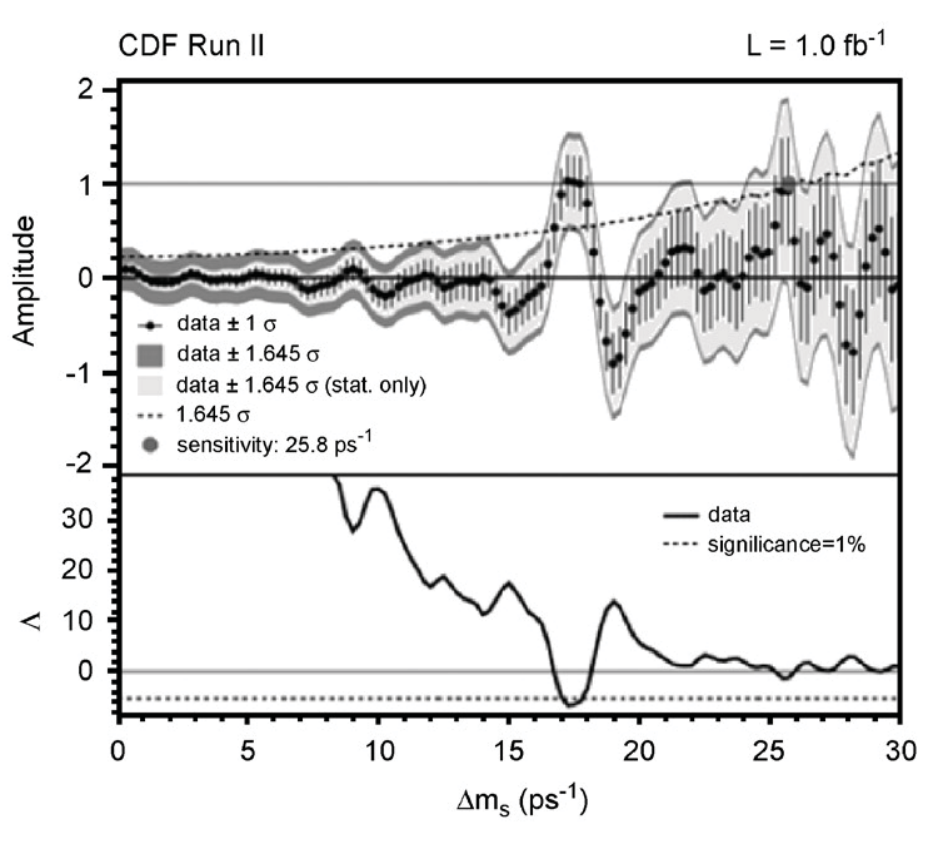

Fig. 1. Illustrations of previous physics measurements where the experiment silicon vertex detector has played a crucial role. Part (a) shows the evolution of the measurement of the $\mathrm{b}$ lifetime during the LEP1 running era (figure courtesy of G. Wilkinson). Part (b) shows the recent signal for $B_{s}$ mixing measured at the CDF experiment [1].

10 years $\left(500 \mathrm{fb}^{-1}\right)$ will be around $3 \times 10^{15} \mathrm{~cm}^{-2}$, approximately $100 \mathrm{Mrad}$, falling by just over a factor 10 at $20 \mathrm{~cm}$.

The upgrade of the LHC to SLHC will pose new challenges. The exact upgrade scenario is yet to be defined but will in all probability occur in a number of phases [2]. The first phase will see a relatively modest increase in luminosity to $2.3 \times 10^{34} \mathrm{~cm}^{-2} \mathrm{~s}^{-1}$, achieved through higher beam currents. Phase 2 would involve a major hardware upgrade to achieve an ultimate luminosity of close to $10^{35} \mathrm{~cm}^{-2} \mathrm{~s}^{-1}$. A final phase could see an upgrade of the beam energy to $14 \mathrm{TeV}$ with the use of new superconducting dipoles. Currently, these phases are dominated by scenarios involving 25 and $50 \mathrm{~ns}$ running.

The charged hadron fluences are illustrated in Fig. 2 for an integrated luminosity of $2500 \mathrm{fb}^{-1}$. At the innermost radius of $4 \mathrm{~cm}$, fluence levels of $1.6 \times 10^{16} \mathrm{~cm}^{-2}$ are expected, and the charged hadrons dominate. At a radius of $20 \mathrm{~cm}$ the level drops by an order of magnitude and is composed of an equal mix of charged and neutral hadrons. From the detector point of view it is the integrated luminosity, rising by a factor of 5 , which will drive the technology choice. Note, however, that with the peak luminosity rising by a factor of 10 , an improved granularity will be needed to achieve the same performance as for the LHC.

The first phase of detector R\&D is focused on short-term modest upgrades, replacing the components closest to the beam which could start to deteriorate. These include the
ATLAS Pixel B layer which will need changing after 2-3 years, the CMS Phase 1 pixel detector and the $\mathrm{LHCb}$ VELO. The second R\&D phase is focused on preparing major changes to the experiment modules and electronics, which could be implemented in synchronisation with the LHC hardware upgrade. Note that other collider environments also pose challenges in terms of irradiation; a 'super B-factory' experiment operating at a luminosity of $10^{36} \mathrm{~cm}^{-2} \mathrm{~s}^{-1}$ will receive 5-10 Mrad yearly dose in the vertex detector region [3].

In this article, we discuss the impact of radiation damage at the SLHC, and the new technologies under development. Moving to the future International Linear Collider (ILC) facility, we describe the contrasting sets of requirements, and the candidate devices which could be used for the innermost vertexing detectors.

\section{Radiation damage}

We discuss here the impact of radiation damage, and the evolution from the LHC to the SLHC environment. Traditionally, the NIEL equivalence [4] has been used to relate the damage caused by impinging particles of various energies and types to that which would be caused by a certain fluence of $1 \mathrm{Mev}$ neutrons $\left(\mathrm{n}_{\mathrm{eq}}\right)$. This has proved to be a powerful tool to normalise the damage expected in complex collider environments to that measured in irradiation programmes. It is, however, known to fail for comparing neutron and charged hadron damage, in certain cases, and care must be taken to distinguish these. At LHC 


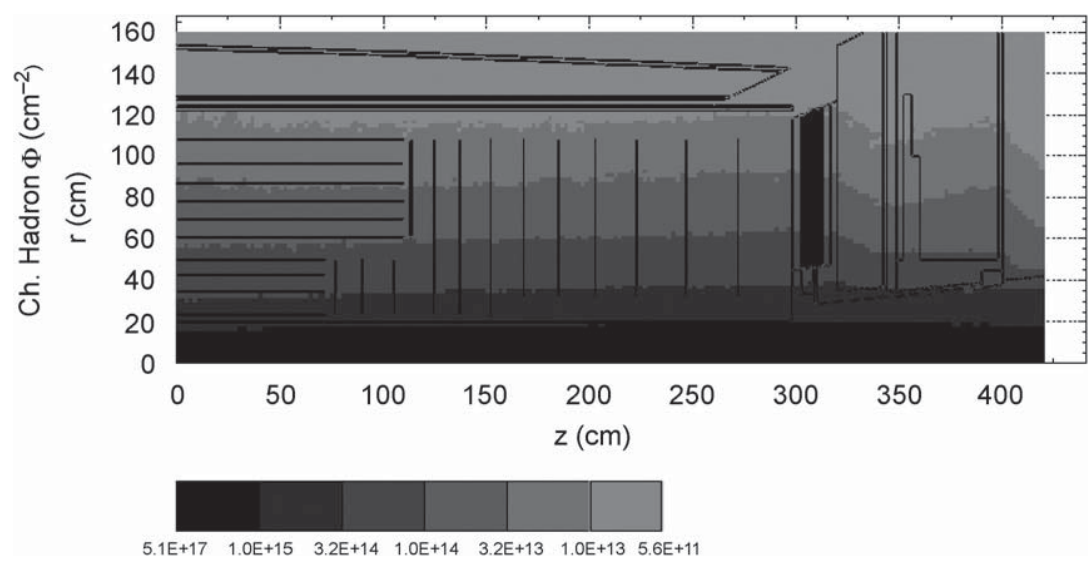

Fig. 2. Charged hadron fluences expected in the SLHC environment, for an integrated luminosity of $2500 \mathrm{fb}^{-1}$. Figure courtesy of M. Huhtinen.

levels, the problems occurring due to radiation damage manifest themselves in two major ways:

- An increase in leakage current, which affects the noise and can make it difficult to deliver bias to the sensors.

- Changes in the effective doping concentration $N_{\text {eff }}$. Charged trapping levels caused by lattice defects typically cause the negative space charge to build up. For classic n-type devices the voltage $V_{\mathrm{fd}}$ necessary to deplete the sensors initially drops, but after doses of typically $5 \times 10^{13} \mathrm{n}_{\mathrm{eq}} \mathrm{cm}^{-2}$ the inversion point is passed, and $V_{\mathrm{fd}}$ rises steeply, eventually passing the point at which the sensor breaks down. In the case of $\mathrm{p}^{+}$-in-n sensors, as the inversion point is passed the depletion region moves from the $\mathrm{p}^{+}$to the $\mathrm{n}^{+}$side, causing significant changes in the electrical properties of the devices.

These effects anneal with time in a strongly temperaturedependent way; there is a need to keep the irradiated sensors below $0{ }^{\circ} \mathrm{C}$, which has significant implications for the operation and maintenance of the detectors. Moving to SLHC fluences, trapping, which gives a loss of signal even at full depletion, starts to play a major role, and the picture becomes more complex. The traditional description of the radiation damage in terms of a single parameter $N_{\text {eff }}$ breaks down, and double junction models have more success in describing the data. As an example, we show in Fig. 3 the measurement of cluster profiles in a row of pixels illuminated by a beam at low incidence angle. It turns out that for fluences of $6 \times 10^{14} \mathrm{n}_{\mathrm{eq}} \mathrm{cm}^{-2}$ the model with constant charge density does not describe the measured charge collection for differently applied bias voltages. A new model [6] has been developed which models the field resulting from a build-up of two dominant types of radiation induced defects, of acceptor and donor type. This results in a large electric field at both sides of the diode (Fig. 4). The parameters of the model have been tuned and a description of the data achieved which gives reasonable agreement across the full range of bias voltages, as well as describing well the expected leakage current levels. The same model has been used to describe transient current technique (TCT) measurements on heavily irradiated diodes, in this case using $\mathrm{MCz}$ silicon [7]. A simulation of the current pulse response has been performed, and it has been shown that movement of charge in the depleted region induces a transient current in the base, which can transfer the injected carriers. These measurements along with others [8] show that, from the point of view of detector operation, full depletion is not a decisive requirement. At high fluences parameters such as charge collection efficiency, charge collection time and signal to noise must be considered as the critical operational parameters. As already demonstrated for $\mathrm{n}^{+}$in-n-type sensors [9], running in underdepleted mode is certainly an option, and can give surprisingly good performances [10].

\section{Radiation at running experiments}

The models of radiation behaviour can now increasingly be started to be compared against long-term operation of silicon sensors at real life experimental facilities. The CERN heavy experiment NA60 has used LHC-type pixel detectors for the first time at a running experiment, operated past the inversion point, after a dose of roughly $10^{13} \mathrm{n}_{\mathrm{eq}} \mathrm{cm}^{-2}$. At CDF and D0, the data collected now exceeds $2 \mathrm{fb}^{-1}$, corresponding to a dose in CDF of $2.5 \mathrm{Mrad}$ at the innermost layer, and $1 \mathrm{Mrad}$ at $2.5 \mathrm{~cm}$. The impact of the radiation can be assessed by analysing the evolution of $V_{\mathrm{fd}}$, by monitoring either the signal as a function of applied voltage, or the noise, which drops at full depletion for the double sided sensors. The results show the expected decrease in $V_{\mathrm{fd}}$, and can be calibrated against the ionising dose measured with Thermo-Luminescent Dosimeters (TLDs), leading to the conclusion that the innermost layers will survive the lifetime of the experiments [11]. 

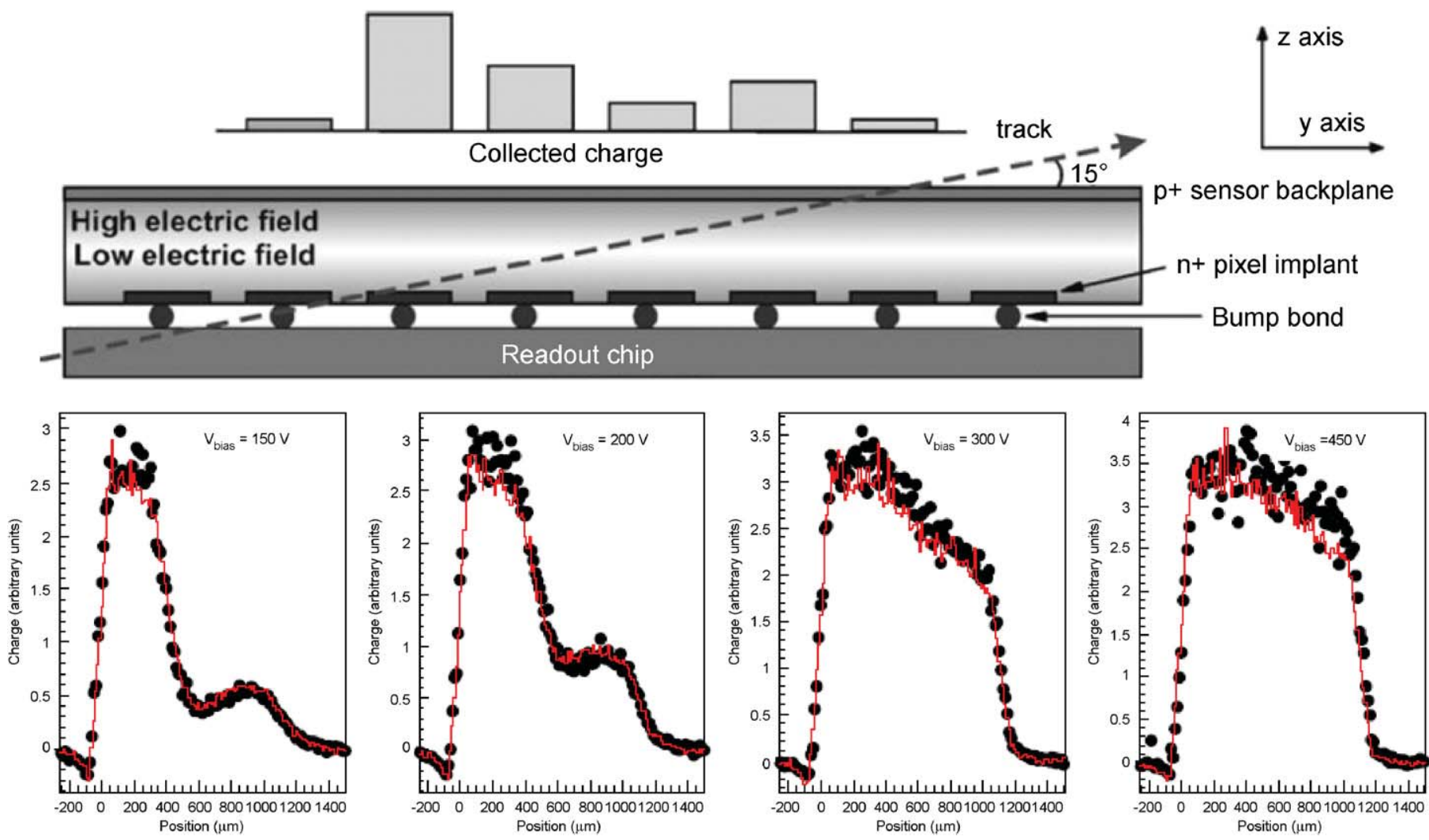

Fig. 3. The grazing angle technique for determining charge collection profiles [5]. The cluster shapes measured in the data for a range of bias voltages (dots) are compared to the tuned simulation (lines) for fluences of $6 \times 10^{14} \mathrm{n}_{\mathrm{eq}} \mathrm{cm}^{-2}$.

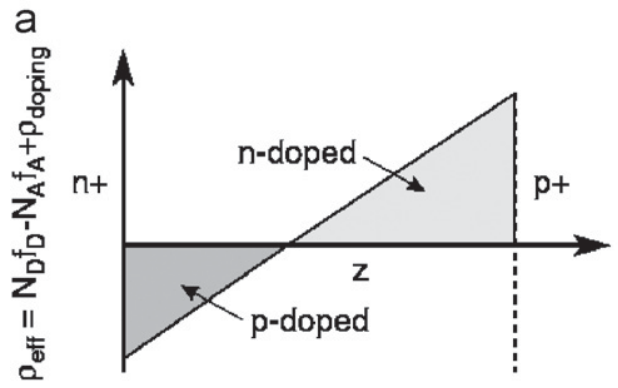

b

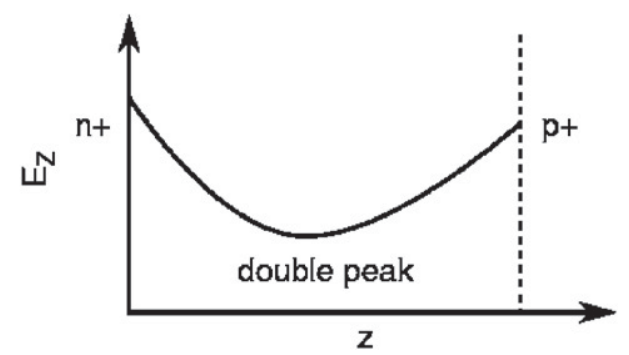

Fig. 4. Illustrative sketch of the $z$-component of the electric field in a heavily irradiated reverse biased silicon detector [5].

\section{New technologies}

As discussed above, new approaches are necessary to cope with the fluences expected at the SLHC, for both the innermost and intermediate radial regions. The CERN RD50 collaboration [12] aims to track the most recent silicon-based discoveries and facilitate research which can see development extended to large-scale segmented devices. The work is grouped into two major categories. 'Defect Engineering' aims to investigate the underlying processes causing the changes in behaviour under irradiation. The problem is then attacked by deliberate modification of the silicon, for instance, by enhancing the oxygen content, or the use of different types of silicon, such as silicon carbide, amorphous silicon and so on. 'Device Engineering' modifies the structures to combat the effects of radiation damage, for instance, by the construction of monolithic devices. It is also possible to enhance detector lifetimes by altering the operational conditions, for example, by going to cryogenic temperatures [13]. Due to lack of space we discuss here only some highlights of this research.

\subsection{P-Type devices}

$\mathrm{n}^{+}$-in-n technology is already the preferred choice for the highly irradiated regions of ATLAS, CMS and LHCb, due to the fast charge collection, and the electric field structure which favours charge collection on the $\mathrm{n}$ side after irradiation. The use of $n^{+}$-in-n structures has the disadvantage that in the early stage of operation there is a 
high field at the back plane junction. They must for this reason be produced with a double sided technology, with guard ring structures implanted in the backside. $\mathrm{n}^{+}$-in- $\mathrm{p}$ structures, where the charge is still collected on $\mathrm{n}^{+}$strips, but these strips are implanted in a p-type bulk, are a promising solution [14]. The main advantage of the p-type devices is that they are simpler to produce and handle due to the single sided processing. The initial behaviour is expected to be similar to that of an $\mathrm{n}^{+}$-in- $\mathrm{n}$ device irradiated just past the inversion point, and post-irradiation all the advantages of the $\mathrm{n}^{+}$-in- $\mathrm{n}$ structures are conserved, with the caveat that for the intermediate irradiation regime the depletion voltages required will be slightly higher.

As shown at this conference [10], p-type short strip devices have been irradiated up to $10 \times 10^{15} \mathrm{n}_{\mathrm{eq}} \mathrm{cm}^{-2}$ and show encouraging charge collection performances. There are even indications that the annealing behaviour, at least with respect to charge collection efficiency, is quite stable, which has good implications for the operational conditions of the detectors. Full scale sensors have been manufactured for $\mathrm{LHCb}$. They comprise strip pitches ranging between 40 and $100 \mu \mathrm{m}$, and make up $2 \%$ of the final detector. The p-type modules (see Fig. 5) were characterised in a testbeam and showed similar signal to noise and bad strip fractions to the n-type modules.

The future challenges of p-type sensor manufacture lie in optimising the strip isolation methods. The various options are illustrated in Fig. 6: the traditional p-stop, where the $\mathrm{n}^{+}$ electrodes are separated by $\mathrm{p}$-type implants, $\mathrm{p}$-spray, which has a uniform p-type application across the surface of the device, or a combination of the two. p-Stop devices can suffer from pre-irradiation micro discharges, while p-spray must be carefully tuned to ensure adequate detector lifetime. This problem applies equally to the $n^{+}$-in- $n$ devices; however, an advantage of $p$ type is that it is immediately possible to optimise for breakdown current post-irradiation. In the context of both the RD50 collaboration and the SMART [16] collaboration, a wide variety of p-type sensors are being manufactured and evaluated.

\subsection{New materials}

Based on the RD48 discovery that $V_{\mathrm{fd}}$ varies more slowly for oxygenated sensors [17], Diffusion Oxygenated Float Zone (DOFZ) silicon, where the oxygen is diffused into the wafers, has been chosen for many experiments. It is particularly appropriate close to the interaction region, where the radiation is dominated by charged hadrons, as it seems to be the case that the benefit is not maintained in the case of neutron irradiation [18]. Many investigations have followed the lines of choosing materials which are

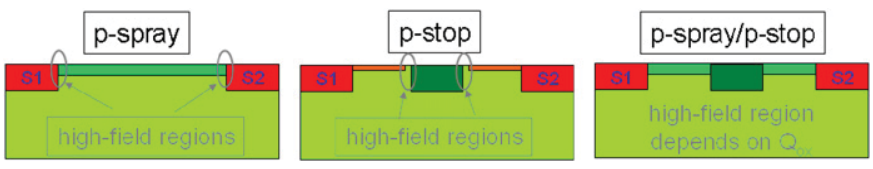

Fig. 6. Diagrammatic illustration of the different options for interelectrode isolation. In the case of p-spray, the interstrip capacitance and breakdown voltage are expected to improve with radiation, while in the case of the p-stop they are more likely to degrade. The combined solution represents a compromise; simulations are necessary for each specific case to find the optimum. Figure courtesy of C. Piemonte.
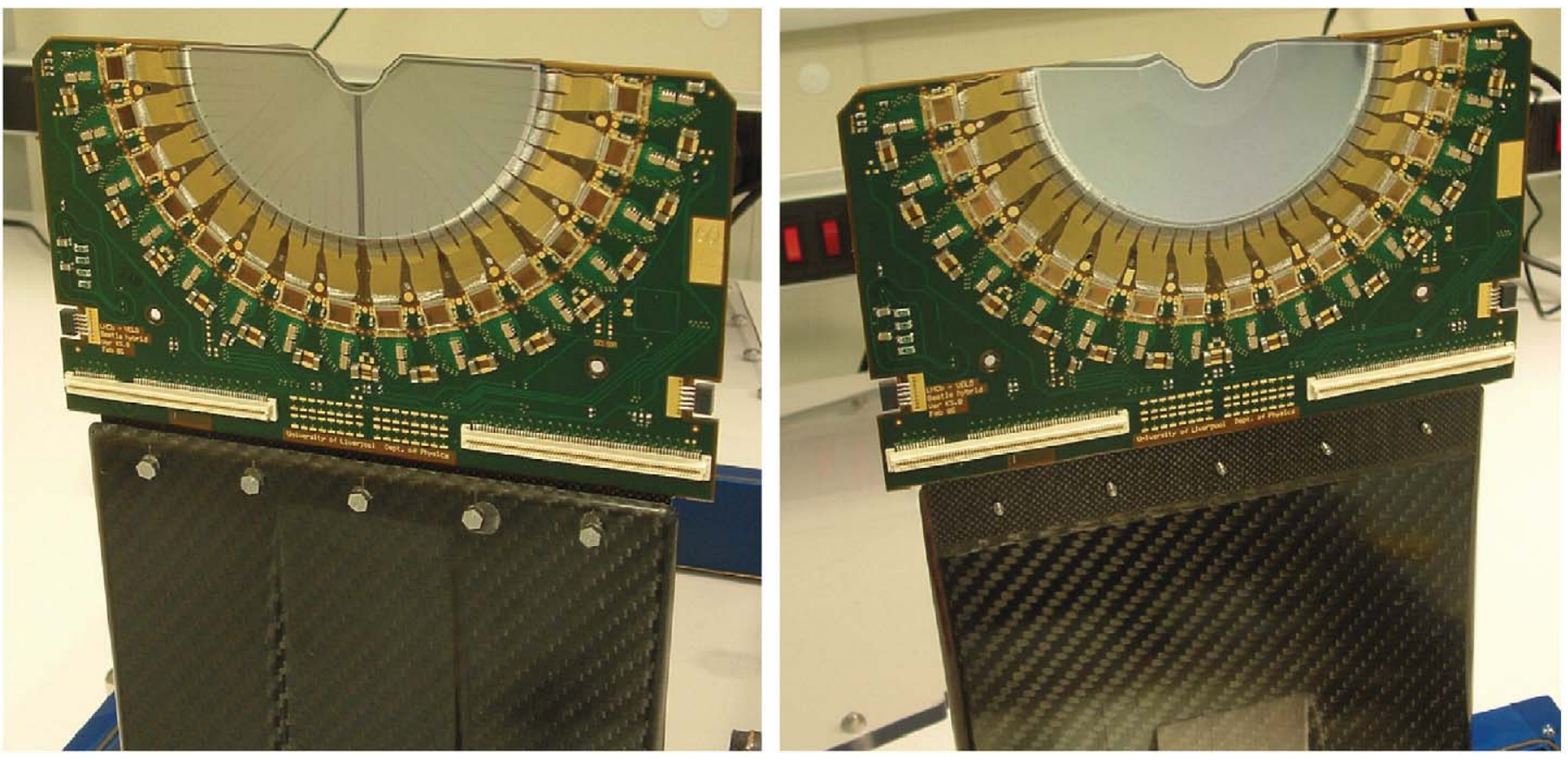

Fig. 5. A module for the LHCb experiment constructed with silicon sensors using $\mathrm{n}^{+}$-in-p technology, manufactured at MICRON [15]. On the left, the front of the module showing the $R$ measuring sensor can be seen, and, on the right, the view of the back of the module showing the Phi measuring sensor. 
naturally rich in oxygen, such as Czochralski $(\mathrm{Cz})$ silicon, which is now available in high-purity samples, or epitaxial silicon, where the CVD silicon grown on the $\mathrm{Cz}$ silicon substrate naturally receives oxygen by diffusion from the bulk. The oxygen concentration in $\mathrm{Cz}$ silicon is at a level of $10^{17}-10^{18} \mathrm{~cm}^{-2}$ and extends uniformly throughout the bulk, in contrast to DOFZ silicon.

Studies on $\mathrm{Cz}$ silicon have confirmed that the slope of the $V_{\mathrm{fd}}$ rise is more modest than for standard and DOFZ samples, and indicate that the annealing behaviour is more stable $[19,20]$. TCT measurements show that for charged hadron irradiation the material does not type invert [21], which makes it attractive from the point of view of a segmented position sensitive device. Irradiation with neutrons shows a more complex picture, and here the double junction model described above gives a better picture [7]. Studies of the charge collection efficiency have shown encouraging results, with a CCE of more than $90 \%$ achieved for a microstrip device with $200 \mathrm{~ns}$ shaping time after a proton irradiation of $3.3 \times 10^{14} \mathrm{n}_{\mathrm{eq}}$ [22]. Simulations have been performed studying the signal to noise as a function of fluence at high levels of irradiation and are shown in Fig. 7. These indicate that the optimal bias voltage shows large variations only in the range $10^{15} \mathrm{n}_{\mathrm{eq}} \mathrm{cm}^{-2}$, with moderate voltages being adequate below this level, and trapping effects dominating above. Comprehensive studies are being carried out in the context of the RD50 and SMART collaborations, with more than 5 institutes actively fabricating particle detectors on $\mathrm{MCz}$ wafers. For a complete review, see Ref. [23].

Samples of epitaxial silicon have also been produced and irradiated to levels of $8 \times 10^{14} \mathrm{n}_{\mathrm{eq}} \mathrm{cm}^{-2}$ [25]. The annealing behaviour shows that in this case it is even beneficial not to cool the device, outside operation. This is of great importance for SLHC operation, where likely scenarios include 100 days operation with beam, with the remainder

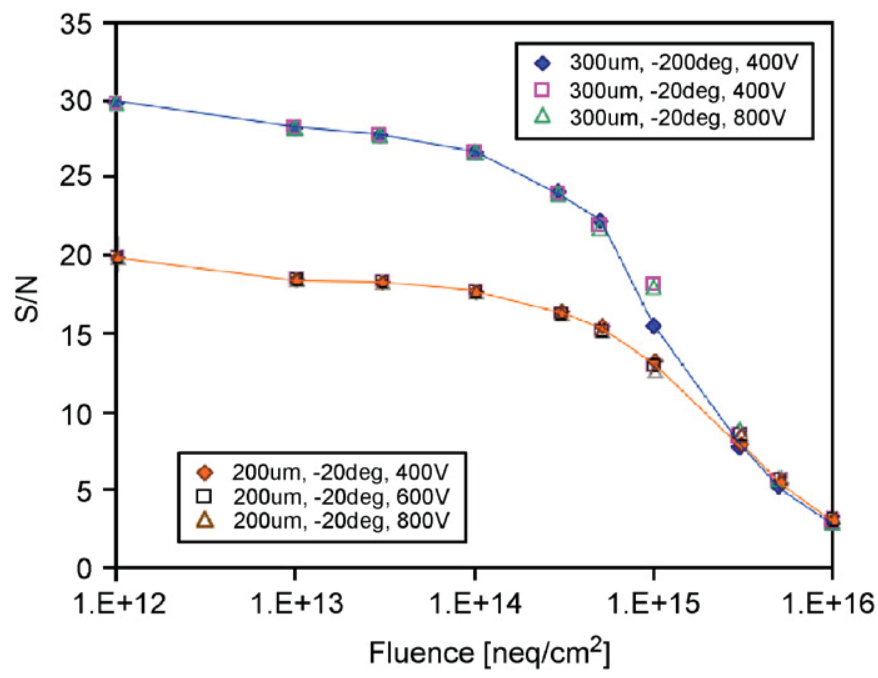

Fig. 7. Simulation of the signal to noise expected for $\mathrm{MCz}$ p-type microstrip detectors, for a strip length of $3 \mathrm{~cm}$ and detector thicknesses of 300 and $200 \mu \mathrm{m}$. From Ref. [24]. split between storage (cooling possible) and maintenance (at room temperature). The CCE measured for a $75 \mu \mathrm{m}$ thick sample after this irradiation level was 3200 electrons (see Fig. 8). This indicates that epitaxial silicon could be considered a good material for performance after high levels of irradiation, whereas before irradiation the small signal is disfavourable.

It has been known for some time that the large band gap and strong atomic bonds of diamond should give fantastic radiation hardness as a detector material [26]. Its low leakage current is a great advantage for cooling issues in the SLHC barrel environment. This, combined with low capacitance and high mobility, leads to good signal to noise, in spite of the high ionisation energy, which gives approximately 2 times less signal for an equivalent silicon radiation length. Polycrystalline samples now give reasonable charge collection and a signal to noise of above 10 for proton irradiation at the level of $1.8 \times 10^{16} \mathrm{p} \mathrm{cm}^{-2}$ (see Fig. 9). Diamond is well established as a detector material, with beam condition monitoring applications at the Tevatron and the LHC [28]. This material is a candidate for the innermost tracking layers at the SLHC, if the cost and procurement issues can be addressed.

\subsection{The $3 D$ detectors}

Another approach to combat radiation damage is to make a radical change in the detector concept. In 3D pixel technology [29], the electrodes penetrate into the bulk instead of lying on the surface of the sensor. This design has the great advantage of decoupling the drift distance, which governs the radiation hardness properties, from the sensor thickness, which dictates the amount of signal collected. The small electrode separation gives intrinsically fast charge collection, and low depletion voltages, along with the possibility of removing dead regions at the

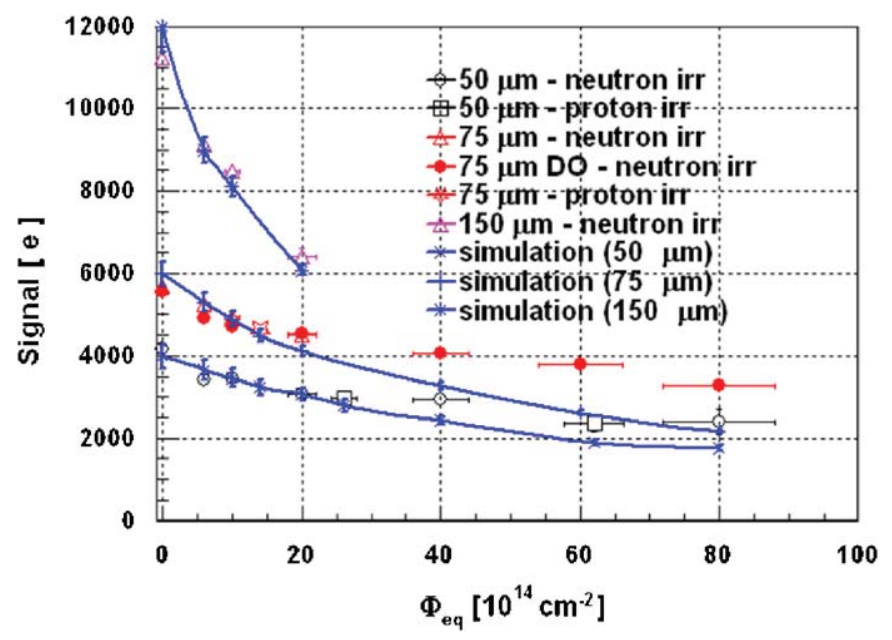

Fig. 8. Charge collection measured with minimum ionising particles for epitaxial layers of various thickness and irradiation levels. The solid lines show the expected signal given by the simulation. Figure courtesy of G. Kramberger. 
a

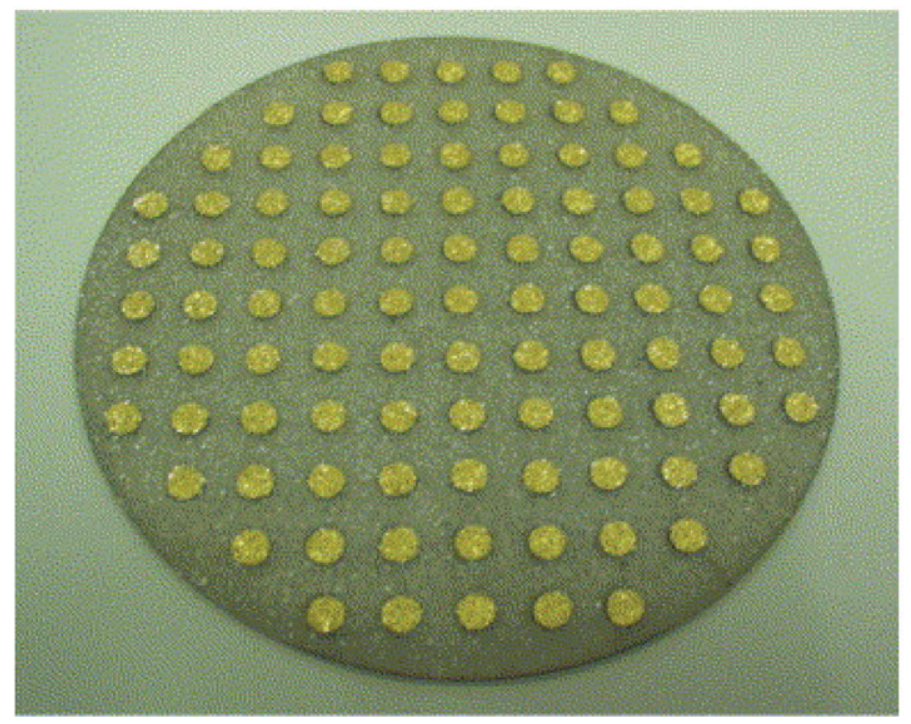

b

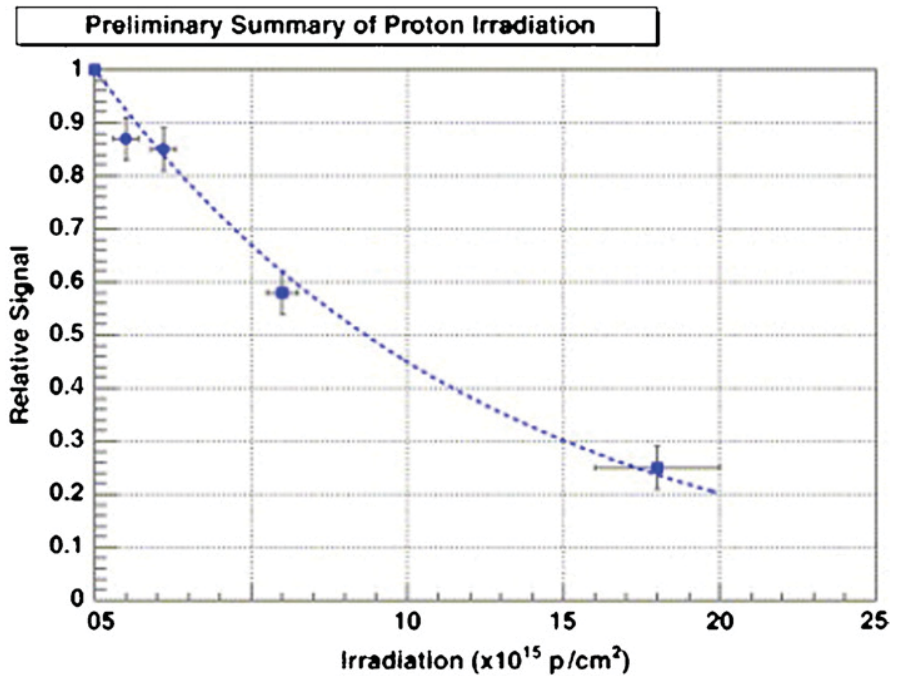

Fig. 9. (a) A pCVD diamond wafer ready for test probing. (b) Signal obtained from a pCVD diamond wafer after irradiation levels up to $1.8 \times 10^{16} \mathrm{p} \mathrm{cm}^{-2}$ [27].

sensor edges. The price to pay is a more complex processing, and the full characterisation of the sensors must be understood.

Recent testbeam results are very encouraging, with efficiencies reaching $98 \%$, and irradiated devices have been characterised with lasers, showing good charge collection efficiency at relatively modest depletion voltages. There are also efforts directed at producing simplified structures, where the electrodes do not reach all the way across the wafer, and so the wafers have similar characteristics but are easier to produce and study. At this conference, position resolved measurements using a laser on such devices have been presented and compared with simulation [30].

\section{Linear Collider applications}

A high-luminosity environment with a radically different set of requirements to hadron machines is provided at the ILC [31]. Whereas at the LHC the focus is on high-rate capability and extreme radiation tolerance, the challenges at the ILC are all about precision, low material and hence ultimate vertexing power. The time structures at the ILC are expected to consist of $950 \mu$ s long trains of approx 2800 bunches with a repetition rate of about $5 \mathrm{~Hz}$. The very short bunch spacing raises the problem of overlapping events; however, the relatively low duty cycle can give new options for the readout.

The physics events at the ILC will have an average jet multiplicity twice that seen at LEP energies, with a very high track density within these jets. The backgrounds can be suppressed with a high solenoidal field, but the occupancy ultimately drives the readout time, which should be achieved in $50 \mu$ s for the innermost layer. The physics signatures generally consist of multijet final states, in which $\mathrm{b}$ and $\mathrm{c}$ tagging is of great importance. In order to cope with these demands, the vertex detector must be pixel based, with a target thickness of $0.1 \% X_{0}$ per layer. The radiation tolerance required is of the order of $360 \mathrm{krad}$ per year. There are a number of candidate technologies being developed for ILC vertex detector applications, all based on the integration of the detecting element with the electronics. We discuss some examples here; for a complete review see Ref. [32].

\subsection{DEPFETs}

An example of such an active pixel sensor is the DEPFET [33], in which the first amplifying transistor is integrated onto the silicon substrate and control is provided by steering chips on the edge of the sensor. As the contact at the backside is an important component of the sensor, it is not immediately apparent how to thin these devices. A possible solution is to use a handle wafer to hold the DEPFET on the previously implanted backside, then to thin and process the top before partially etching away the wafer, leaving a perforated frame. In this way a thickness of $50 \mu \mathrm{m}$ has been achieved at the pixels, giving an estimated average layer thickness of $0.11 \% X_{0}$. The wafer processing does not degrade the leakage currents, which are measured at the level of $\approx 100 \mathrm{pA} \mathrm{cm}^{-2}$ [34].

The possible layout of a DEPFET vertex detector innermost layer at the ILC is illustrated in Fig. 10. The steering chips lie along the length of the wafers, and the readout chips on either end. In a recent testbeam a signal to noise of 110 was registered for a $450 \mu \mathrm{m}$ thick device, and the radiation hardness has been demonstrated above 1 Mrad. Future developments will concentrate on improving the noise, and increasing the readout speed by improving the zero suppression performance. 


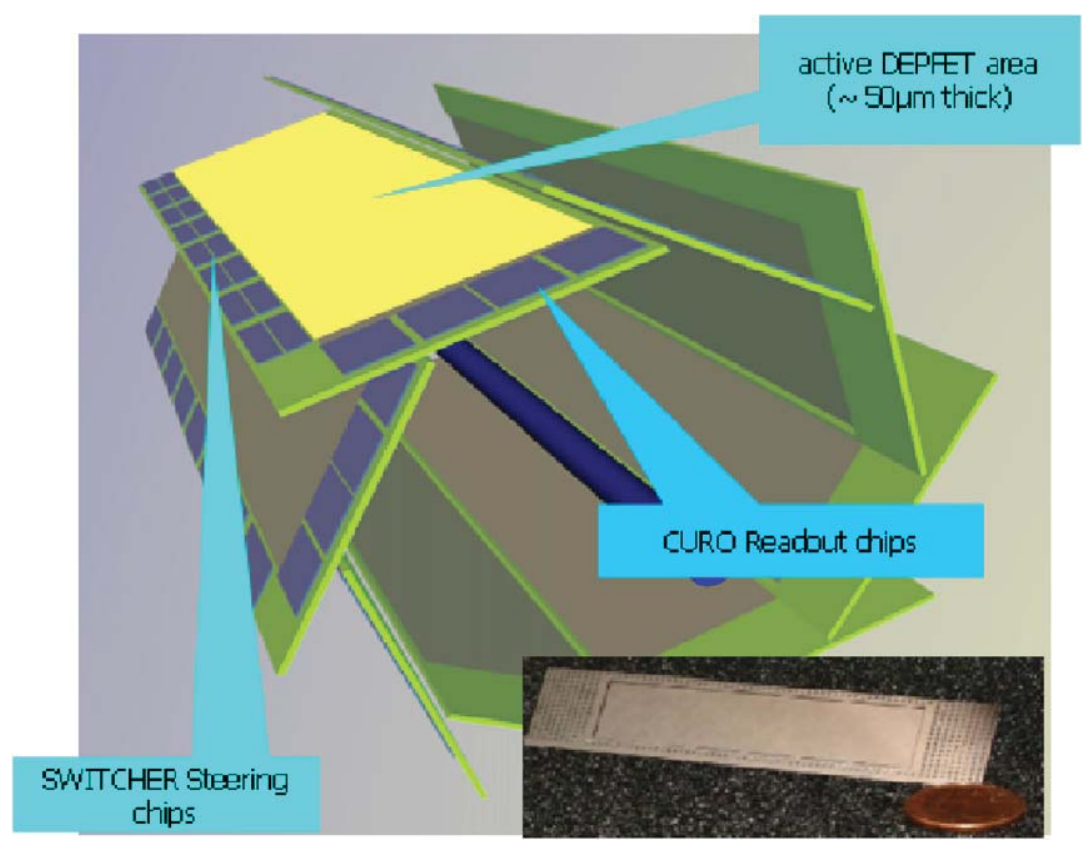

Fig. 10. A possible layout of DEPFET sensors for the innermost layer of the vertex detector of the Linear Collider, showing the position of the steering chips along the edges of the sensors and the readout chips at the end. Inset: a photograph of a successfully thinned sensor, with a pixel thickness of $50 \mu \mathrm{m}$ and a frame thickness of $350 \mu \mathrm{m}[34]$.

\subsection{Monolithic Active Pixel Sensors (MAPS)}

MAPS [35] are an alternative solution, consisting of a thin epitaxial layer where the charge is collected, superimposed with a pixel array of n-wells. This concept has undergone very rapid development, with much effort concentrated on the MIMOSA [36] chip, which has completed at least 15 full cycles of chip design since 1999 . The perfect charge sharing leads to spectacular position resolution, with values of a few microns regularly demonstrated in testbeams.

In principle the modern submicron electronics should be able to withstand high doses of ionising radiation, but it is important to be sure that the charge collection from the epitaxial layer is preserved and that the leakage current does not increase the detector noise. The chips have been exposed to non-ionising radiation levels of $10^{11} \mathrm{n}_{\mathrm{eq}} \mathrm{cm}^{-2}$ (equivalent to 10 years operation at the ILC) and subsequently showed no obvious increase in noise, and an efficiency of $99.97 \%$. A separate batch was exposed to ionising radiation levels of $10^{13} \mathrm{e} \mathrm{cm}^{-2}$, and showed a slight increase in noise and an efficiency of $99.4 \%$ [37]. Fig. 11 shows the signal measured with a ${ }^{55} \mathrm{Fe}$ source before and after irradiation with $1 \mathrm{Mrad}$ $\mathrm{X}$-rays.

A sensor adapted to the upgrade of the STAR vertex detector, which must be able to operate at $+40^{\circ} \mathrm{C}$, is being developed, and the first prototype is under test. For use at the ILC additional R\&D is needed to reach the required readout speed, and a suitable column parallel readout architectures with on-pixel CDS are being developed [39].

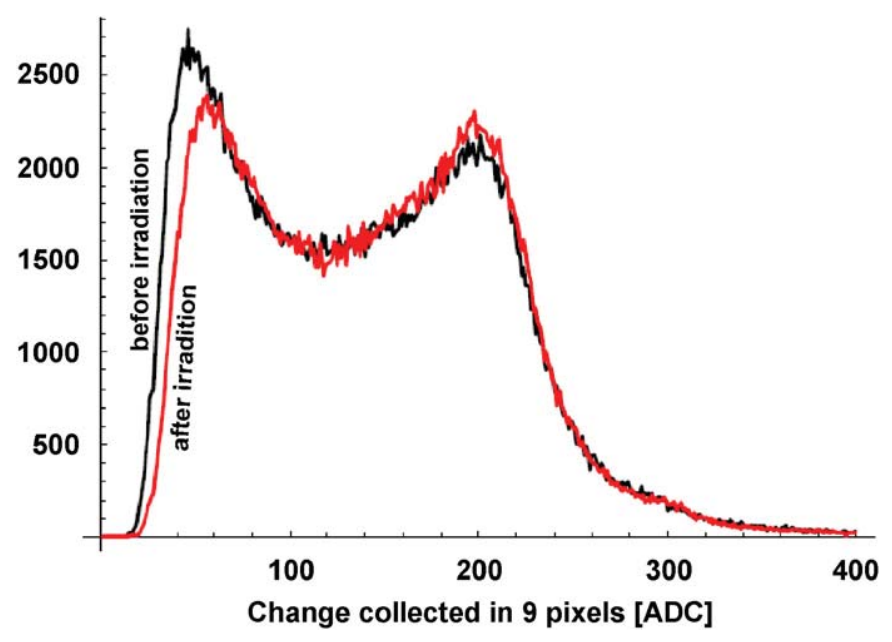

Fig. 11. Tolerance of a MAPS prototype, SUCCESSOR1, to ionising radiation doses of $1 \mathrm{Mrad}$. The charge collection in a cluster of 9 pixels is shown before and after irradiation [38].

\subsection{Charge Coupled Devices (CCDs)}

CCDs [40] are a well-established baseline option for the ILC vertex detector design. The 307 Mpixel SLD vertex detector [41] proved that this technology could be used to build and operate a large area detector with high precision and gain uniformity. For implementation at ILC the next major steps must address the readout speed and radiation hardness issues.

The basic operational principle of the CCD is similar to the MAPS in that the charge is collected from the epitaxial layer, but in this case it is clocked out of the 


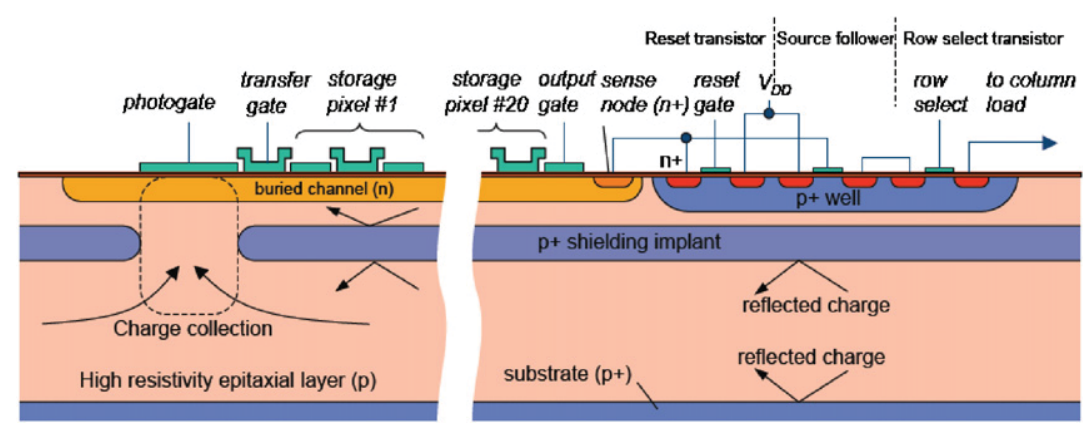

Fig. 12. Principle of operation of the ISIS sensor [43].

sensor before the first amplification stage. A major innovation developed for the ILC design is a move from the traditional serial register readout, where the charges from each readout column are moved towards a readout circuit on the corner, to a column parallel readout, where the readout chip receives analogue signals from all columns in parallel. The concept has already been demonstrated, and the next generation of CCDs in development uses a busline free design, in order to achieve the goal of a $50 \mathrm{MHz}$ clock propagation (a factor of 10 more than that at SLD). In parallel, aggressive mechanical designs have been introduced to reduce significantly the material present. A prototype using $20 \mu \mathrm{m}$ thin silicon attached with glue pillars to $1.5 \mathrm{~mm}$ of silicon carbide foam shows good mechanical and thermal stability, and the range of $0.1 X_{0}$ radiation length per layer seems approachable [42].

In a new development, the CCD concept has been extended to the In-situ Storage Image Sensor (ISIS). This sensor aims to combat the beam related RF pickup, which could disrupt the signal if charge to voltage conversions occur during the bunch train. The principle of operation is illustrated in Fig. 12. Each pixel contains CCD-style charge storage cells, which can be implemented in CMOS or CCD technology. The charge is collected at the photogate and transferred to a storage pixel during the bunch train. During each bunch train 20 transfers operate, and the readout occurs during the $200 \mathrm{~ms}$ quiet period after a bunch train, during which a clocking speed of $1 \mathrm{MHz}$ should be adequate. A proof-of-principle device has been operated and the design of a small pixel ISIS2 is scheduled for 2007 [43].

\subsection{Silicon on Insulator (SOI)}

Development of a monolithic pixel detector based on SOI technology has been carried out by the SUCIMA [44] and SOIPIX [45] collaborations. The process fabricates circuitry on top of a silicon-oxide layer on the surface of a high-resistivity silicon substrate. This should lead in principle to a fast and low-power operation, due to the fact that the transistors are isolated from the substrate and from each other. This very new technology has already been shown to record signals from minimum ionising particles in a testbeam. More recent developments are based on CMOS technology, which should in the long term provide good industrial support for the development of SOI technology as a radiation sensor.

\section{Conclusions}

The challenges of operation at future high-luminosity colliders are being addressed by a wide range of developments in semiconductor detector technology. These developments build on the achievements of the currently operating devices as well as those in the commissioning stage. Such systems have benefited from the reliable partnerships built up with industry, as well as the rad hard $0.25 \mu \mathrm{m}$ CMOS readout systems for the electronics. There have been many steps forward in the understanding of the mechanisms of radiation damage, at the microscopic and macroscopic level, and models describing the properties of highly irradiated detectors have shown great success. This paper has only described in part some of the new ideas in this very vibrant field, many of which have been discussed in more detail at this conference. The development of new materials and structures for the LHC is continuing apace, while at the same time intense activity is seeing the development of ultra-low-mass pixel systems dedicated for the ILC.

\section{Acknowledgement}

I would like to thank the organisers for the opportunity to present this paper at this fascinating and superbly run conference, and look forward to the exciting developments in detector technologies to be announced at future editions.

\section{References}

[1] A. Abulencia, et al., hep-ex/0606027v1.

[2] O. Bruning, in: CMS Workshop for SLHC, CERN, February 2004; D. Denegri, in: SLHC/VLHC Magnet Workshop, CERN, June 2006.

[3] M. Barbero, et al., IEEE Trans Nucl. Sci. NS-52(4) 1187.

[4] A. Vasilescu, in: DESY-Proceedings-1998-02, 1998.

[5] V. Chiochia, et al., physics $/ 0411143$.

[6] V. Eremin, E. Verbitskaya, Z. Li, Nucl. Instr. and Meth. A 476 (2002) 556.

[7] E. Verbitskaya, et al., Nucl. Instr. and Meth. A 557 (2006) 538. 
[8] G. Casse, et al., Nucl. Instr. and Meth. A 426 (1999) 140; D. Menichelli, et al., Nucl. Instr. and Meth. A 426 (1999) 135; A. Castaldini, et al., J. Appl. Phys. 92 (2002) 2013.

[9] P.P. Allport, et al., Nucl. Instr. and Meth. 450 (2000) 297; K. Borer, et al., Nucl. Instr. and Meth. 440 (2000) 17.

[10] G. Casse, et al., Response to minimum ionising particles of p-type substrate silicon microstrip detectors irradiated with neutrons to LHC upgrade doses, these proceedings; M. Lozano, et al., Ultimate limits for the radiation hardness of silicon strip detectors for sLHC, these proceedings.

[11] A. Pronko, The CDF silicon detector: performance and longevity, these proceedings.

[12] R. Bates, RD50 Status: developing radiation tolerant materials for ultra radiation-hard tracking detectors, these proceedings.

[13] J. Haerkonen, The cryogenic transient current technique (C-TCT) measurement setup of CERN RD39 Collaboration, these proceedings.

[14] M. Artuso, Silicon sensors implemented on p-type substrates for high radiation resistance application, in: VERTEX06, The 15th International Workshop on Vertex Detectors, Perugia, September 2006, Nucl. Instr. and Meth., submitted for publication.

[15] Micron Semiconductor Ltd., Lancing Business Park, West Sussex, UK.

[16] H.F.-W. Sadrozinski, et al., Nucl. Instr. and Meth., in press.

[17] 3rd RD48 Status Report, CERN LHCC 2000-009, Available from 〈http://rd48.web.cern.ch/rd48/〉.

[18] A. Ruzin, et al., IEEE Trans. Nucl. Sci. NS-46 (1999) 1310; D. Bisello, et al., IEEE Trans. Nucl. Sci. NS-49 (2002) 1027.

[19] E. Tuominen, J. Haerkoenen, et al., IEEE Trans. Nucl. Sci. NS-50 (2003) 1942.

[20] G. Pellegrini, et al., Nucl. Instr. and Meth. A 552 (2005) 27.

[21] A. Bates, M. Moll, Nucl. Instr. and Meth. A 555 (2005) 113; S. Dittongo, et al., Nucl. Instr. and Meth. A 546 (2005) 300; A. Bates, Nucl. Instr. and Meth. A 569 (2006) 73.

[22] M. Bruzzi, et al., in: The Sixth International Hiroshima Symposium 2006, Carmel, CA, USA, 2006.

[23] A. Messineo, Czochralski silicon sensors: status of development, in: VERTEX06, The 15th International Workshop on Vertex Detectors, Perugia, September 2006, Nucl. Instr. and Meth., submitted for publication.

[24] M. Bruzzi, H.F.-W. Sadrozinski, A. Seiden, Comparing radiation tolerant materials and devices for ultra rad-hard tracking detectors, in: STD6, Carmel, CA September 11-16, Nucl. Instr. and Meth., submitted for publication.

[25] G. Lindstroem, in: 10th European Symposium on Semiconductor Detectors, University of Hamburg, Wildbad Kreuth, 12-16th June 2005.
[26] R. Wallny, Status of diamond detectors and their high energy physics application, in: VERTEX06, The 15th International Workshop on Vertex Detectors, Perugia, September 2006, Nucl. Instr. and Meth., submitted for publication.

[27] W. Adam, et al., Nucl. Instr. and Meth. A 565 (2006) 278.

[28] I. Dolenc, presented at this conference.

[29] S. Parker, et al., University of Hawaii Internal Report UH 511-83996, 1996.

[30] S. Eckert, et al., Signal and charge collection efficiency of a p-type STC 3D-detector irradiated to sLHC-fluences, read out with $40 \mathrm{MHz}$, these proceedings.

[31] J. Mnich, Detectors for a linear collider, these proceedings.

[32] In: ILC VTX Workshop at Ringberg Castle, May 2006, 〈http:// www.hll.mpg.de/lca/ringberg//.

[33] J. Kemmer, G. Lutz, Nucl. Instr. and Meth. A 253 (1987) 365.

[34] P. Fischer, et al., Progress towards a large area, thin DEPFET detector module, in: VERTEX06, The 15th International Workshop on Vertex Detectors, Perugia, September 2006, Nucl. Instr. and Meth., submitted for publication.

[35] W. Dulinski, presented at this conference; L. Servoli et al., Test of a MAPS realized in standard non-epitaxial CMOS 0.18 micron technology, these proceedings; R. Turchetta, et al., Nucl. Instr. and Meth. A 560 (2006) 139.

[36] For more information see: 〈http://ireswww.in2p3.fr/ires/recherche/ capteurs $/\rangle$.

[37] M. Deveaux, et al., Nucl. Instr. Meth. A 512 (2003) 71;

M. Deveaux et al., Charge collection properties of monolithic active pixel sensors (MAPS) irradiated with non-ionising radiation, in: Winter Proceedings of the Sixth RESMDD-Conference 2006, Florence, Nucl. Inst. Meth., submitted for publication.

[38] M. Deveaux, et al., Nucl. Instr. and Meth. A 552 (2005) 118.

[39] G. Gaycken, et al., Nucl. Instr. and Meth. A 560 (2006) 44.

[40] C.J.S. Damerell, Nucl. Instr. and Meth. A 541 (2005) 178.

[41] J. Brau, IEEE Trans. Nucl. Sci. 43 (3) (1996) 1107.

[42] A. Nomerotski, Optimisation of vertex detector for international linear collider, in: VERTEX06, The 15th International Workshop on Vertex Detectors, Perugia, September 2006, Nucl. Instr. and Meth., submitted for publication.

[43] S. Worm, Progress with vertex detector sensors for the linear collider, in: VERTEX06, The 15th International Workshop on Vertex Detectors, Perugia, September 2006, Nucl. Instr. and Meth., submitted for publication.

[44] W. Kucewicz, et al., Nucl. Instr. and Meth. A 541 (2005) 172.

[45] T. Tsuboyama, et al., R\&D of a monolithic pixel sensor based on $0.15 \mu \mathrm{m}$ fully depleted SOI technology, these proceedings. 